\title{
On the Spectrum of the Derangement Graph
}

\author{
Paul Renteln* \\ Department of Physics \\ California State University \\ San Bernardino, CA 92407 \\ and \\ Department of Mathematics \\ California Institute of Technology \\ Pasadena, CA 91125 \\ prenteln@csusb.edu
}

Submitted: Apr 10, 2007; Accepted: Nov 1, 2007; Published: Nov 28, 2007

Mathematics Subject Classifications: 05C25, 05C50, 05E05, 05E10

\begin{abstract}
We derive several interesting formulae for the eigenvalues of the derangement graph and use them to settle affirmatively a conjecture of $\mathrm{Ku}$ regarding the least eigenvalue.
\end{abstract}

Keywords: Cayley Graph, Least Eigenvalue, Derangement Graph, Symmetric Group, Symmetric Function Theory, Complete Symmetric Factorial Functions, Shifted Schur Functions

\section{Introduction}

Let $G$ be a finite group and $S \subseteq G$ a symmetric subset of generators $\left(s \in S \Rightarrow s^{-1} \in S\right)$ satisfying $1 \notin S$. The Cayley graph $\Gamma(G, S)$ has the elements of $G$ as its vertices, and two elements $u, v \in G$ are joined by an edge provided $v u^{-1}=s$ for some $s \in S$. ${ }^{1}$ It is clear that $\Gamma(G, S)$ is regular of vertex degree $|S|$. Let $S_{n}$ be the symmetric group of permutations of $X=\{1,2, \ldots, n\}$, and let $\mathcal{D}_{n}:=\left\{\sigma \in S_{n}: \sigma(x) \neq x, \forall x \in X\right\}$ denote

\footnotetext{
${ }^{*}$ I would like to thank Cheng $\mathrm{Ku}$ for bringing his conjecture to my attention and for many stimulating discussions, David Wales for pointing out a discrepancy in an earlier draft, Rick Wilson and the mathematics department of the California Institute of Technology for their kind hospitality, and the referee for suggesting several improvements in the exposition.

${ }^{1}$ The condition $1 \notin S$ precludes loops, while the symmetry condition allows us to consider the graph as being undirected.
} 
the derangements on $X$, namely the set of fixed point free permutations of $S_{n}$. (Note that $\mathcal{D}_{n}$ is symmetric in the above sense, as the inverse of a derangement is a derangement.) We call $\Gamma_{n}:=\Gamma\left(S_{n}, \mathcal{D}_{n}\right)$ the derangement graph on $X$. Much is known about this graph:

- $\Gamma_{n}$ is connected $(n>3)$. This follows because every permutation can be written as the product of adjacent transpositions $(k, k+1)$, and these, in turn, can be expressed as the product of the two derangements $(1,2, \ldots, n)^{2}$ and $(n, n-1, \ldots, 1)^{2}(k, k+1)$. (If $n=3$ this fails because the product of two odd permutations is even.) Thus, for $n>3$ the derangements generate $S_{n}$, which means that every vertex of $\Gamma_{n}$ can be reached from the identity. To ensure $\Gamma_{n}$ is connected we therefore assume $n \geq 4$ in all that follows.

- $\Gamma_{n}$ is Hamiltonian. This was first observed by Eggleton and Wallis [7] and subsequently by others (see, e.g., [18]).

- $\alpha\left(\Gamma_{n}\right)=(n-1)$ !, where $\alpha$ is the independence number. This was first proved by Deza and Frankl [5], who also observed that the bound is achieved by a coset of the stabilizer of a point. Cameron and $\mathrm{Ku}$ [3] (and, independently, Larose and Malvenuto [12]) showed that these are the only such maximum independent sets. (See also [9].)

- $\omega\left(\Gamma_{n}\right)=n$, where $\omega$ is the clique number, because a maximum clique in $\Gamma_{n}$ is just a Latin square of size $n$.

- $\chi\left(\Gamma_{n}\right)=n$, where $\chi$ is the chromatic number. This follows from a result of Godsil. We say a Cayley graph $\Gamma(G, S)$ is normal if $S$ is closed under conjugation. Godsil shows ([8], Corollary 7.1.3) that for any normal Cayley graph, $\chi(\Gamma)=\omega(\Gamma)$ if $\alpha(\Gamma) \omega(\Gamma)=|V(\Gamma)|$, where $|V(\Gamma)|$ is the number of vertices of $\Gamma$. The generating set $\mathcal{D}_{n}$ of $\Gamma_{n}$ is a union of conjugacy classes, because a derangement is just a permutation with no cycles of length one, and cycle type is preserved under conjugation. $\Gamma_{n}$ has $n$ ! vertices, so the claim follows.

Now recall that, for any regular graph of degree $k$ with $N$ vertices, the independence number satisfies the Delsarte-Hoffman bound

$$
\alpha \leq N \frac{-\eta}{k-\eta}
$$

where $\eta$ is the least eigenvalue of the adjacency matrix of the graph. Graphs in which equality holds have several interesting properties. For example, in such graphs we have equality between $\alpha$ and the Shannon capacity of the graph. (For an extensive discussion of the Delsarte-Hoffman bound and its implications, see [14].) For the derangement graph $N=n !$ and $k=D_{n}:=\left|\mathcal{D}_{n}\right|$, so we get

$$
\eta \leq \frac{-D_{n}}{n-1}
$$


This prompted Cheng $\mathrm{Ku}$ to make the following

Conjecture 1.1. [See, e.g., [11]] The least eigenvalue of the adjacency matrix of the derangement graph is given by

$$
\eta=\frac{-D_{n}}{n-1}
$$

The main objective of this work is to provide several interesting formulae for the eigenvalues of the derangement graph and to prove Conjecture 1.1. We begin by recalling a result due to Diaconis and Shahshahani on the eigenvalues of a normal Cayley graph. This leads us to a discussion of the characters of the symmetric group. Using a result of Stanley we relate these to symmetric function theory and compute a rough bound on the eigenvalues. We then employ the factorial symmetric functions of Chen and Louck (which

are related to the shifted symmetric functions of Okounkov and Olshanski) to derive a remarkable recurrence formula for the eigenvalues of $\Gamma_{n}$. This is the critical tool we need to prove the conjecture.

\section{The Standard Representation}

The following theorem for the eigenvalues of a normal Cayley graph is due to Diaconis and Shahshahani [6] (for an earlier related result, see [1]; for the version below, see [17]):

Theorem 2.1. Let $A$ be the adjacency matrix of a normal Cayley graph $\Gamma(G, S)$. Then the eigenvalues of $A$ are given by

$$
\eta_{\chi}=\frac{1}{\chi(1)} \sum_{s \in S} \chi(s),
$$

where $\chi$ ranges over all the irreducible characters of $G$. Moreover, the multiplicity of $\eta_{\chi}$ is $\chi(1)^{2}$.

Recall that a partition $\lambda$ of $n$, written $\lambda \vdash n$ or $|\lambda|=n$, is a weakly decreasing sequence $\left(\lambda_{1}, \lambda_{2}, \ldots, \lambda_{\ell}\right)$ such that $\sum_{i} \lambda_{i}=n$. Its length is $\ell$ and each $\lambda_{i}$ is a part of the partition. Partitions are represented by Ferrers diagrams:
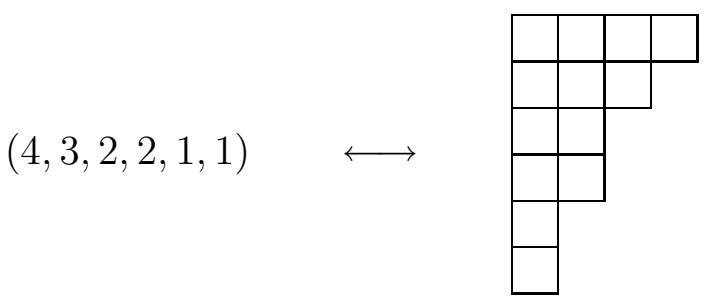

and are also written using multiplicity notation

$$
(4,3,2,2,1,1) \quad \longleftrightarrow \quad 4^{1} 3^{1} 2^{2} 1^{2} .
$$


As is well known (see, e.g., $[10,13,20])$ the irreducible characters $\chi_{\lambda}$ of $S_{n}$ are indexed by partitions $\lambda \vdash n$. Also, as the cycle type of a permutation of $S_{n}$ is just the partition whose parts are the cycle lengths, the conjugacy classes of $S_{n}$ are also labeled by partitions.

The standard representation of $S_{n}$ corresponding to the partition $\lambda=(n-1,1)$ plays an important role in the sequel. It is constructed as follows. Let $V$ be an $n$ dimensional inner product space with orthonormal basis $\left(e_{1}, e_{2}, \ldots, e_{n}\right)$. Then $S_{n}$ acts on $V$ by permuting each vector

$$
\sigma\left(e_{i}\right)=e_{\sigma(i)}
$$

and extending by linearity. One says that $V$ affords the defining representation of $S_{n}$. It is clear that $S_{n}$ leaves fixed the one dimensional subspace $U$ generated by the vector $\sum_{i} e_{i}$, so $U$ affords the trivial representation of $S_{n}$ (which is clearly irreducible). The orthogonal complement $W=U^{\perp}$ also affords an irreducible representation of dimension $n-1$, namely the standard representation, and we have the equivariant decomposition

$$
V=U \oplus W
$$

As characters are additive on direct sums, it follows that

$$
\chi_{W}=\chi_{V}-\chi_{U} .
$$

$\chi_{V}(\sigma)$ just counts the number of fixed points of $\sigma$, so

$$
\chi_{W}(\sigma)=\#\{\text { fixed points of } \sigma\}-1 .
$$

From (2.1) and Theorem 2.1 the eigenvalue of the derangement graph corresponding to the standard representation $W$ is thus

$$
\eta_{W}=\frac{1}{\chi_{W}(1)} \sum_{\sigma \in \mathcal{D}_{n}} \chi_{W}(\sigma)=\frac{-D_{n}}{n-1} .
$$

This is precisely the conjectured least eigenvalue.

In the table below we illustrate the truth of Conjecture 1.1 for the derangement graph $\Gamma_{6}$ by summing the characters of $S_{6}$ over the derangements. Notice that the standard representation yields the least eigenvalue $\left(\eta_{5^{1} 1^{1}}=-53\right) .{ }^{2}$

\footnotetext{
${ }^{2}$ The first half of the table is taken from [10] with corrections for the minor typographical errors therein. The second half is obtained by pointwise multiplication of the entries in the first half by the alternating character.
} 


\begin{tabular}{|r|c|c|c|c|c|c|c|c|c|c|c|c|}
\hline Class & $1^{6}$ & $2^{1} 1^{4}$ & $2^{2} 1^{2}$ & $2^{3}$ & $3^{1} 1^{3}$ & $3^{1} 2^{1} 1^{1}$ & $3^{2}$ & $4^{1} 1^{2}$ & $4^{1} 2^{1}$ & $5^{1} 1^{1}$ & $6^{1}$ & $\eta_{\lambda}$ \\
\hline \# Elts & 1 & 15 & 45 & 15 & 40 & 120 & 40 & 90 & 90 & 144 & 120 & \\
\hline $6^{1}$ & 1 & 1 & 1 & 1 & 1 & 1 & 1 & 1 & 1 & 1 & 1 & +265 \\
\hline $5^{1} 1^{1}$ & 5 & 3 & 1 & -1 & 2 & 0 & -1 & 1 & -1 & 0 & -1 & -53 \\
\hline $4^{1} 2^{1}$ & 9 & 3 & 1 & 3 & 0 & 0 & 0 & -1 & 1 & -1 & 0 & +15 \\
\hline $4^{1} 1^{2}$ & 10 & 2 & -2 & -2 & 1 & -1 & 1 & 0 & 0 & 0 & 1 & +13 \\
\hline $3^{2}$ & 5 & 1 & 1 & -3 & -1 & 1 & 2 & -1 & -1 & 0 & 0 & -11 \\
\hline $3^{1} 2^{1} 1^{1}$ & 16 & 0 & 0 & 0 & -2 & 0 & -2 & 0 & 0 & 1 & 0 & -5 \\
\hline $3^{1} 1^{3}$ & 10 & -2 & -2 & 2 & 1 & 1 & 1 & 0 & 0 & 0 & -1 & -5 \\
\hline $2^{3}$ & 5 & -1 & 1 & 3 & -1 & -1 & 2 & 1 & -1 & 0 & 0 & +7 \\
\hline $2^{2} 1^{2}$ & 9 & -3 & 1 & -3 & 0 & 0 & 0 & 1 & 1 & -1 & 0 & +5 \\
\hline $2^{1} 1^{4}$ & 5 & -3 & 1 & 1 & 2 & 0 & -1 & -1 & -1 & 0 & 1 & +1 \\
\hline $1^{6}$ & 1 & -1 & 1 & -1 & 1 & -1 & 1 & -1 & 1 & 1 & -1 & -5 \\
\hline
\end{tabular}

\section{The Eigenvalues of the Derangement Graph}

Next we derive an explicit formula for the eigenvalues of the derangement graph. We assume the reader has some familiarity with symmetric function theory, but for completeness we recall a few facts here (for more details, see e.g., [10, 13, 20]). Consider the ring $\mathbb{Z}\left[x_{1}, x_{2}, \ldots, x_{n}\right]$ of all polynomial functions in $n$ variables over the integers. The symmetric group acts by permuting variables, and the invariant polynomials form the ring of symmetric functions

$$
\Lambda_{n}=\mathbb{Z}\left[x_{1}, x_{2}, \ldots, x_{n}\right]^{S_{n}} .
$$

There are many bases for $\Lambda_{n}$. In what follows we will use two: the complete (homogeneous) symmetric functions and the Schur functions. Given a partition $\lambda=\left(\lambda_{1}, \lambda_{2}, \ldots, \lambda_{n}\right)$, the complete symmetric function $h_{\lambda}$ is defined by

$$
h_{\lambda}:=h_{\lambda_{1}} h_{\lambda_{2}} \cdots h_{\lambda_{n}}
$$

where

$$
h_{k}:=\sum_{i_{1}+i_{2}+\cdots+i_{n}=k} x_{1}^{i_{1}} x_{2}^{i_{2}} \cdots x_{n}^{i_{n}}
$$

and $i_{j} \in \mathbb{Z}_{\geq 0}$ for $j=1, \ldots, n$.

There are many equivalent ways to define the Schur functions. The combinatorial definition is as follows. A semistandard Young tableau of shape $\lambda$ is a Ferrers diagram of $\lambda$ in which the boxes are filled with numbers that weakly increase across rows and strictly 
increase down columns. For example,

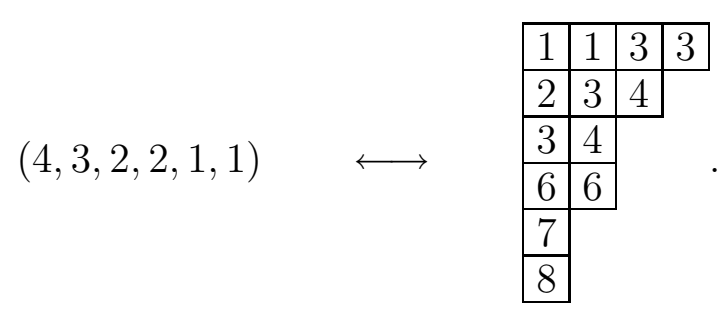

The type of $T$ is a vector giving the multiplicities of each entry in the tableau. In the above example, type $(T)=(2,1,4,2,0,2,1,1)$. Associated to each tableau is the monomial denoted $x^{T}$, defined by raising each variable to its corresponding entry in the type vector. For the above example

$$
x^{T}=x_{1}^{2} x_{2} x_{3}^{4} x_{4}^{2} x_{6}^{2} x_{7} x_{8}
$$

A semistandard Young tableau $T$ is standard if type $(T)=\overbrace{(1,1, \ldots, 1)}^{n \text { times }}$, which means that it is filled with the numbers from 1 to $|\lambda|$.

This construction admits a slight generalization. Let $\nu \subseteq \lambda$ (i.e., $\nu_{i} \leq \lambda_{i}$ for all $i$ ). A skew semistandard Young tableau of shape $\lambda / \nu$ and type $\alpha$ is obtained by subtracting the boxes of the Ferrers shape of $\nu$ from those of $\lambda$ and filling in the boxes as before. For example, if $\lambda=(4,3,2,2,1,1), \nu=(3,2,1)$, and $\alpha=(1,0,2,1,1,1,1)$, one such tableau is
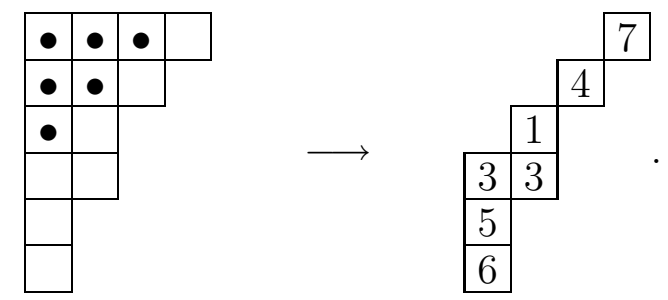

The tableau monomials are defined as before. Then the skew Schur function of shape $\lambda / \nu$ is

$$
s_{\lambda / \nu}\left(x_{1}, x_{2}, \ldots, x_{n}\right)=\sum_{T} x^{T}
$$

where the sum extends over all skew semistandard Young tableau of shape $\lambda / \nu$. Although it is not obvious from this definition, $s_{\lambda / \nu}$ is a symmetric function (see, e.g., [20], Theorem 7.10.2, p. 311). If $\nu=\emptyset$ then $s_{\lambda}$ is the Schur function of shape $\lambda$.

The canonical (or Hall) inner product on $\Lambda_{n}$ can be defined by the requirement that the Schur functions comprise an orthonormal basis:

$$
\left(s_{\lambda}, s_{\nu}\right)=\delta_{\lambda, \nu}
$$

It can be shown $([20]$, Eq. 7.61$)$ that

$$
\left(s_{\lambda}, h_{\nu}\right)=K_{\lambda, \nu}
$$


where $K_{\lambda, \nu}$ is the Kostka number, namely the number of semistandard Young tableau of shape $\lambda$ and type $\nu$.

Following Stanley we define

$$
d_{\lambda}:=\sum_{s \in \mathcal{D}_{n}} \chi_{\lambda}(s)
$$

where $\chi_{\lambda}$ is the irreducible character of the symmetric group corresponding to the partition $\lambda$. Stanley shows that this function admits a nice expansion in terms of Schur functions:

Theorem 3.1. [[20], Exercise 7.63, p. 519]

$$
\sum_{\lambda \vdash n} d_{\lambda} s_{\lambda}=\sum_{k=0}^{n}(-1)^{n-k}(n)_{k} h_{k 1^{n-k}}
$$

where $(n)_{k}=n(n-1) \cdots(n-k+1)$ is the falling factorial function.

Taking inner products of both sides of (3.2) with $s_{\lambda}$ gives

$$
d_{\lambda}=\sum_{k=0}^{n}(-1)^{n-k}(n)_{k} K_{\lambda, k 1^{n-k}} .
$$

It is clear that $K_{\lambda, k 1^{n-k}}=f^{\lambda / k}$ where $f^{\lambda / \mu}$ is the number of standard Young tableau of skew shape $\lambda / \mu$, because the type $k 1^{n-k}$ means that there are $k$ ones in the Young diagram, and these are necessarily all in the top row. The remaining entries must all be distinct. By (3.1) and Theorem 2.1 the eigenvalues of the derangment graph can be written

$$
\eta_{\lambda}:=\frac{d_{\lambda}}{f^{\lambda}}
$$

because (see, e.g., [20], Equation 7.79) the dimension $\chi_{\lambda}(1)$ of the irreducible representation corresponding to the irreducible character $\chi_{\lambda}$ is simply the number of standard Young tableau of shape $\lambda$. Hence we get

Theorem 3.2. The eigenvalues of the derangement graph are given by

$$
\eta_{\lambda}=\sum_{k=0}^{n}(-1)^{n-k}(n)_{k} \frac{f^{\lambda / k}}{f^{\lambda}}
$$

A more explicit formula for $\eta_{\lambda}$ can be obtained by using Frobenius' formula for the number of standard Young tableau of skew shape ([20], Cor. 7.16.3, p. 344):

$$
f^{\lambda / \mu}=|\lambda / \mu| ! \operatorname{det}\left(\frac{1}{\left(\lambda_{i}-\mu_{j}-i+j\right) !}\right)_{i, j=1}^{n},
$$


where $\lambda \vdash n$ (and $1 / x !=0$ if $x<0$ ). The number of skew standard Young tableau of shape $\lambda / k$ is thus

$$
f^{\lambda / k}=(n-k) !\left|\begin{array}{ccccc}
\frac{1}{\left(\lambda_{1}-k\right) !} & \frac{1}{\left(\lambda_{1}+1\right) !} & \frac{1}{\left(\lambda_{1}+2\right) !} & \cdots & \frac{1}{\left(\lambda_{1}+\ell-1\right) !} \\
\frac{1}{\left(\lambda_{2}-k-1\right) !} & \frac{1}{\lambda_{2} !} & \frac{1}{\left(\lambda_{2}+1\right) !} & \cdots & \frac{1}{\left(\lambda_{2}+\ell-2\right) !} \\
\frac{1}{\left(\lambda_{3}-k-2\right) !} & \frac{1}{\left(\lambda_{3}-1\right) !} & \frac{1}{\lambda_{3} !} & \cdots & \frac{1}{\left(\lambda_{3}+\ell-3\right) !} \\
\vdots & \vdots & \vdots & \ddots &
\end{array}\right|_{\ell \times \ell}
$$

where $\ell$ is the length of $\lambda$. Following the usual convention we define the shifted partition $\mu$ associated to $\lambda$

$$
\mu_{i}:=\lambda_{i}+\ell-i
$$

In terms of $\mu$ we can write

$$
f^{\lambda / k}=(n-k) !\left|\begin{array}{ccccc}
\frac{1}{\left(\mu_{1}-\ell-k+1\right) !} & \frac{1}{\left(\mu_{1}-\ell+2\right) !} & \frac{1}{\left(\mu_{1}-\ell+3\right) !} & \cdots & \frac{1}{\mu_{1} !} \\
\frac{1}{\left(\mu_{2}-\ell-k+1\right) !} & \frac{1}{\left(\mu_{2}-\ell+2\right) !} & \frac{1}{\left(\mu_{2}-\ell+3\right) !} & \cdots & \frac{1}{\mu_{2} !} \\
\frac{1}{\left(\mu_{3}-\ell-k+1\right) !} & \frac{1}{\left(\mu_{3}-\ell+2\right) !} & \frac{1}{\left(\mu_{3}-\ell+3\right) !} & \cdots & \frac{1}{\mu_{3} !} \\
\vdots & \vdots & \vdots & \ddots &
\end{array}\right|_{\ell \times \ell} .
$$

Factoring out the terms in the last column gives

$$
f^{\lambda / k}=\frac{(n-k) !}{\prod_{i} \mu_{i} !}\left|\begin{array}{ccccc}
\left(\mu_{1}\right)_{\ell+k-1} & \left(\mu_{1}\right)_{\ell-2} & \left(\mu_{1}\right)_{\ell-3} & \cdots & 1 \\
\left(\mu_{2}\right)_{\ell+k-1} & \left(\mu_{2}\right)_{\ell-2} & \left(\mu_{2}\right)_{\ell-3} & \cdots & 1 \\
\left(\mu_{3}\right)_{\ell+k-1} & \left(\mu_{3}\right)_{\ell-2} & \left(\mu_{3}\right)_{\ell-3} & \cdots & 1 \\
\vdots & \vdots & \vdots & \ddots &
\end{array}\right|_{\ell \times \ell} .
$$

$(x)_{n}$ is a monic polynomial of degree $n$ in $x$, so using column operations on the last $\ell-1$ columns we get

$$
f^{\lambda / k}=\frac{(n-k) !}{\prod_{i} \mu_{i} !}|M(\mu)|,
$$

where

$$
M(\mu):=\left(\begin{array}{ccccc}
\left(\mu_{1}\right)_{\ell+k-1} & \mu_{1}^{\ell-2} & \mu_{1}^{\ell-3} & \cdots & 1 \\
\left(\mu_{2}\right)_{\ell+k-1} & \mu_{2}^{\ell-2} & \mu_{2}^{\ell-3} & \cdots & 1 \\
\left(\mu_{3}\right)_{\ell+k-1} & \mu_{3}^{\ell-2} & \mu_{3}^{\ell-3} & \cdots & 1 \\
\vdots & \vdots & \vdots & \ddots &
\end{array}\right)_{\ell \times \ell} .
$$

Combining Theorem 3.2, Equations (3.10) and (3.11), and the well-known degree formula $(c f .,[10],(11.6))$

$$
f^{\lambda}=\frac{n !}{\prod_{i} \mu_{i} !} \prod_{i<j}\left(\mu_{i}-\mu_{j}\right)
$$

yields 
Theorem 3.3. The eigenvalues of the derangement graph are given by

$$
\eta_{\lambda}=\sum_{k=0}^{n}(-1)^{n-k} \frac{|M(\mu)|}{\prod_{i<j}\left(\mu_{i}-\mu_{j}\right)} .
$$

\section{Derangement Numbers and an Approximation Scheme}

One approach to evaluating (3.13) is to sum the determinants. To this end, for all $m \geq 0$ we define a shifted derangement number

$$
b(r ; m):=\sum_{k=0}^{r}(-1)^{r-k}(r)_{k+m} .
$$

The ordinary derangement number $D_{r}$ is $b(r ; 0)$ (see, e.g., [21], p. 67).

Lemma 4.1. The derangement numbers satsify the following properties:

i. The first six derangement numbers are $D_{0}=1, D_{1}=0, D_{2}=1, D_{3}=2, D_{4}=9$, and $D_{5}=44$.

ii. $D_{n}=[n ! / e]$, where $[x]$ is the nearest integer to $x$. In particular, the derangement numbers are monotonic increasing for $n \geq 1$.

iii. For $n \geq 1$ the derangement numbers satisfy the following recursions:

$$
D_{n}=n D_{n-1}+(-1)^{n}
$$

and

$$
D_{n}=(n-1)\left(D_{n-1}+D_{n-2}\right) .
$$

Proof. These properties all follow easily from the definition of $D_{n}$.

Theorem 4.2. The eigenvalues of the derangement graph are given by

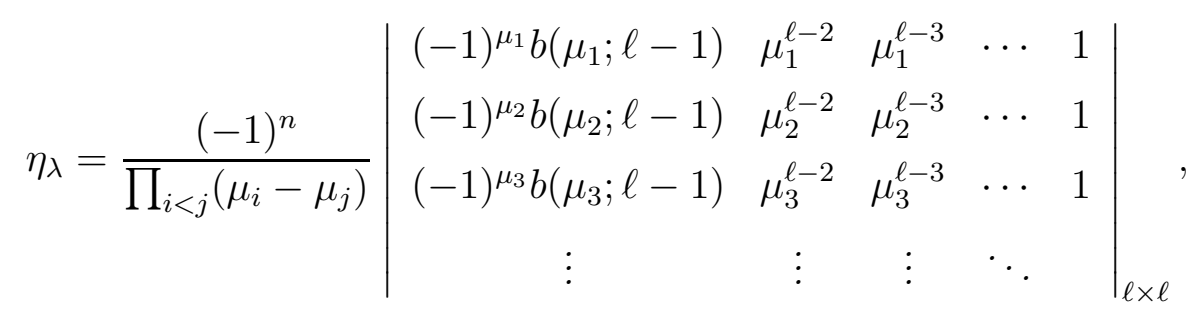

where $\mu$ is defined as in (3.7).

Proof. This follows immediately from (3.13), (4.1), and the multilinearity of the determinant. 
We can use Theorem 4.4 to approximate $\eta_{\lambda}$. First we need

Lemma 4.3. The shifted derangement number satisfies

$$
b(r ; m)=(-1)^{m}(r)_{m} D_{r-m} .
$$

Proof. Use (4.1) and the fact that

$$
(r)_{k+m}=\frac{r !}{(r-m-k) !}=\frac{r !}{(r-m) !} \frac{(r-m) !}{(r-m-k) !}=(r)_{m}(r-m)_{k} .
$$

From Lemma 4.1 and Lemma 4.3 we get

$$
b(r ; m)=(-1)^{m} \frac{r !}{(r-m) !} D_{r-m} \approx(-1)^{m} \frac{r !}{e},
$$

whence

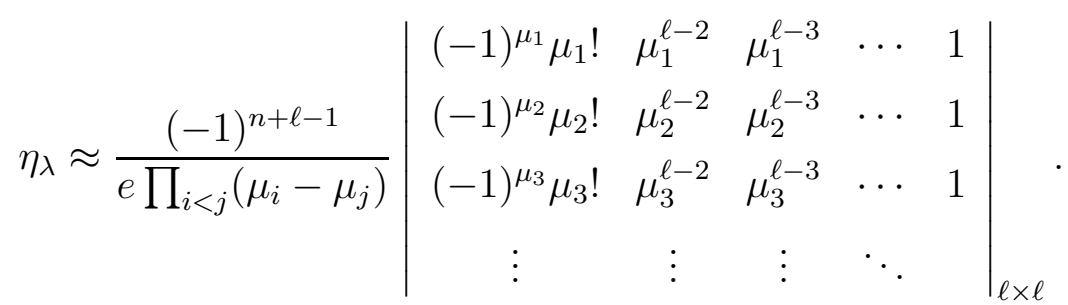

When $\mu_{1}$ is much greater than $\mu_{2}, \mu_{3}, \ldots$ the first term in the Laplace expansion of the determinant by the first column dominates the other terms, and we have

$$
\begin{aligned}
\eta_{\lambda} & \approx \frac{(-1)^{n+l-1}}{e}(-1)^{\mu_{1}} \frac{\mu_{1} !}{\left(\mu_{1}-\mu_{2}\right)\left(\mu_{1}-\mu_{3}\right) \cdots\left(\mu_{1}-\mu_{l}\right)} \\
& \approx \frac{1}{e}(-1)^{n+l-1+\mu_{1}} \frac{\mu_{1} !}{\mu_{1}^{l}} .
\end{aligned}
$$

This gives a rough estimate for the eigenvalues when $\lambda_{1} \gg \lambda_{2}$.

\section{Complete Factorial Symmetric Functions}

Instead of summing the determinants, we investigate the structure of the summands more carefully. Begin again with (3.13). It follows from results of Chen and Louck [4] that the summands can be expressed in terms of what they call complete factorial symmetric functions $w_{k}\left(z_{1}, z_{2}, \ldots, z_{\ell}\right) .{ }^{3}$ We recall some of their results here. The key is their Lemma 2.1 (which they attribute to Verde-Star [22]):

\footnotetext{
${ }^{3}$ In [4] Chen and Louck also define what they call factorial Schur functions, which were generalized by Macdonald [13], and subsequently generalized further by Okounkov and Olshanski [16] to what they called shifted Schur functions. The summands are special cases of shifted Schur functions. We will point out some connections of our results to those of Okounkov and Olshanski as we proceed.
} 
Lemma 5.1. The divided difference of the falling factorial function is

$$
\frac{(x)_{m+1}-(y)_{m+1}}{x-y}=\sum_{0 \leq k \leq m}(x)_{k}(y-k-1)_{m-k} .
$$

Using this lemma iteratively gives ([4], Proposition 2.2 and Equation 2.5)

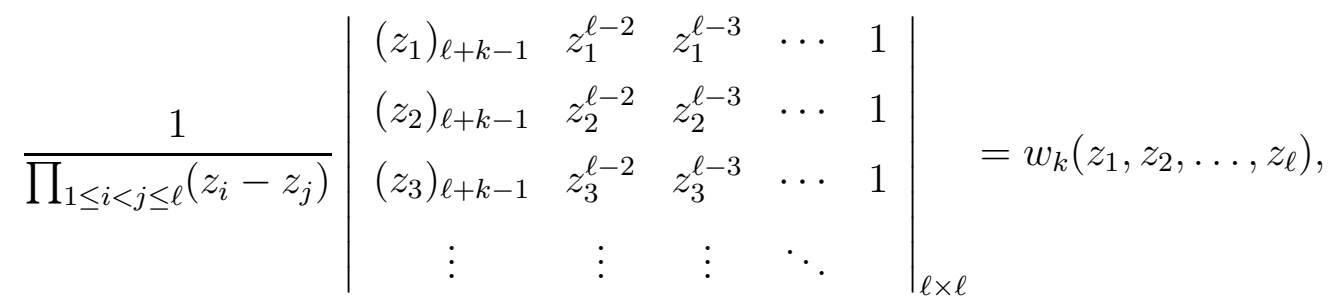

where

$$
w_{k}\left(z_{1}, z_{2}, \ldots, z_{\ell}\right)=\sum_{i_{1}+i_{2}+\cdots+i_{\ell}=k} \prod_{1 \leq j \leq \ell}\left(z_{j}-i_{1}-i_{2}-\cdots-i_{j-1}-j+1\right)_{i_{j}}
$$

or, equivalently,

$$
w_{k}\left(z_{1}, z_{2}, \ldots, z_{\ell}\right)=\sum_{1 \leq i_{1} \leq i_{2} \leq \cdots \leq i_{k} \leq \ell}\left(z_{i_{1}}-i_{1}+1\right)\left(z_{i_{2}}-i_{2}\right)\left(z_{i_{3}}-i_{3}-1\right) \cdots\left(z_{i_{k}}-i_{k}-k+2\right) .
$$

Observe that these functions, while symmetric, are inhomogeneous. ${ }^{4,5}$ They are related to the ordinary complete symmetric functions by

$$
w_{k}\left(z_{1}, z_{2}, \ldots, z_{\ell}\right)=h_{k}\left(z_{1}, z_{2}, \ldots, z_{\ell}\right)+\text { lower order terms. }
$$

Of particular importance for our purposes are the following two properties of the complete factorial symmetric functions. The first is a stabilization property: ${ }^{6}$

\section{Theorem 5.2.}

$$
w_{k}\left(z_{1}, z_{2}, \ldots, z_{\ell-1}, 0\right)=w_{k}\left(z_{1}, z_{2}, \ldots, z_{\ell-1}\right)
$$

Proof. This follows directly from (5.1) by factoring out the product $z_{1} z_{2} \ldots z_{\ell-1}$ from both the numerator and denominator.

The second is a recurrence property:

Theorem 5.3. [[4], Proposition 2.4]

$$
w_{k}\left(z_{1}, z_{2}, \ldots, z_{\ell}\right)=w_{k}\left(z_{2}-1, z_{3}-1, \ldots, z_{\ell}-1\right)+z_{1} w_{k-1}\left(z_{1}-1, z_{2}-1, \ldots, z_{\ell}-1\right) .
$$

We will use both of these results in the next section.

\footnotetext{
${ }^{4}$ The symmetry follows immediately from (5.1) and the well known fact that the quotient of an alternating polynomial by the Vandermonde determinant is a symmetric polynomial.

${ }^{5}$ Okounkov and Olshanski also define what they refer to as complete shifted functions $h_{k}^{*}\left(x_{1}, x_{2}, \ldots, x_{\ell}\right)$, but these are neither symmetric nor homogeneous. They become symmetric when reexpressed in terms of the shifted variables $z_{i}=x_{i}-i+$ constant. By picking the constant judiciously we get $h_{k}^{*}\left(x_{1}, x_{2}, \ldots, x_{\ell}\right)=w_{k}\left(z_{1}, z_{2}, \ldots, z_{\ell}\right)$. Notice that the relationship between the two sets of variables is precisely the relationship between $\lambda$ and $\mu$ (cf., (3.7)).

${ }^{6}$ This result is implicit in the work of [4] and also follows from the corresponding stabilization property of the shifted Schur functions given in [16].
} 


\section{A Recurrence Relation for the Eigenvalues of the Derangement Graph}

Comparing Theorem 3.3 and (5.1) we obtain

Theorem 6.1. The eigenvalues of the derangement graph are given by

$$
\eta_{\lambda}=\sum_{k=0}^{n}(-1)^{n-k} w_{k}\left(\mu_{1}, \mu_{2}, \ldots, \mu_{\ell}\right),
$$

where $\mu$ is the shifted partition associated to $\lambda$ (defined in (3.7)) and $n=|\lambda|$.

Remark 6.2. It follows from Theorem 6.1 that the spectrum of the derangement graph is integral. This result can also be shown directly from Theorem 2.1 by observing that central characters are algebraic integers (see, e.g., [10] (3.2) or [19], Corollary 1 of Proposition $16, p$. 52) and that the characters of the symmetric group are integral valued.

From Theorem 3.2 and Theorem 6.1 we obtain the useful identification ${ }^{7}$

$$
w_{k}\left(\mu_{1}, \mu_{2}, \ldots, \mu_{\ell}\right)=(n)_{k} \frac{f^{\lambda / k}}{f^{\lambda}} .
$$

Next we combine Theorem 5.3 and Theorem 6.1 to derive a powerful recurrence relation for the eigenvalues of the derangement graph. To this end we observe that the $w_{k} \mathrm{~s}$ satisfy a vanishing property: 8

Theorem 6.3. $w_{k}\left(\mu_{1}, \mu_{2}, \ldots, \mu_{\ell}\right)=0$ whenever $k>\lambda_{1}$.

Proof. From (3.7), $\mu_{i}=\lambda_{i}+\ell-i$. Thus, if $k>\lambda_{1}$ then $k+\ell-1>\lambda_{1}+\ell-1=\mu_{1}$. But, by construction, the parts $\lambda_{i}$ are weakly decreasing, so the parts $\mu_{i}$ are strongly decreasing. Hence $k+\ell-1>\mu_{i}$ for all $i$, and the entire first column of the matrix in the numerator in (5.1) (with $\left.z_{i}=\mu_{i}\right)$ vanishes.

Remark 6.4. In the light of (6.2) the vanishing theorem merely formalizes the intuitive idea that we cannot remove more than $\lambda_{1}$ boxes from the first row of a Young tableau.

To state the main result of this section we need some terminology. To any tableau of shape $\lambda$ we may assign $x y$-coordinates to each of the boxes by defining the upper-leftmost box to be $(1,1)$, with the $x$ axis increasing to the right and the $y$ axis increasing downwards. Then the hook through the box $(x, y)$ is the union of the boxes $\left(x^{\prime}, y\right)$ and $\left(x, y^{\prime}\right)$, where $x^{\prime} \geq x$ and $y^{\prime} \geq y$. We will call the hook through the box $(1,1)$ the principal hook (of $\lambda$ ). By abuse of notation, we let $h$ denote either the principal hook itself or its cardinality. (Its meaning will be clear from context.) We also let $\lambda-h$ denote the partition

\footnotetext{
${ }^{7}$ This is a special case of Theorem 8.1 in [16].

${ }^{8}$ This is a special case of the vanishing theorem of Okounkov and Olshanski ([16], Theorem 3.1).
} 
obtained from $\lambda$ by removing the principal hook. We call the first column of the tableau of shape $\lambda$ the principal ladder (of $\lambda$ ), and define $\lambda-1$ as the partition obtained from $\lambda$ by removing its principal ladder. (The latter notation makes more sense if one thinks of $\lambda-1$ as the vector subtraction $\left(\lambda_{1}, \lambda_{2}, \ldots, \lambda_{\ell}\right)-(1,1, \ldots, 1)$.) By successively removing the principal hooks and ladders of $\lambda$ we obtain the key result.

Theorem 6.5. For any partition $\lambda$ the eigenvalues of the derangement graph satisfy the following recurrence:

$$
\eta_{\lambda}=(-1)^{h}\left(\eta_{\lambda-h}+(-1)^{\lambda_{1}} h \eta_{\lambda-1}\right)
$$

with initial condition $\eta_{\emptyset}=1$.

Proof. By Theorem 6.1 and the vanishing theorem we can write

$$
\eta_{\lambda}^{\prime}=\sum_{k=0}^{\infty}(-1)^{k} w_{k}\left(\mu_{1}, \mu_{2}, \ldots, \mu_{\ell}\right)
$$

where

$$
\eta_{\lambda}^{\prime}:=(-1)^{|\lambda|} \eta_{\lambda}
$$

Using (5.5) in (6.4) yields

$$
\eta_{\lambda}^{\prime}=\sum_{k=0}^{\infty}(-1)^{k}\left[w_{k}\left(\mu_{2}-1, \mu_{3}-1, \ldots, \mu_{\ell}-1\right)+\mu_{1} w_{k-1}\left(\mu_{1}-1, \mu_{2}-1, \ldots, \mu_{\ell}-1\right)\right] .
$$

From (3.7) we know that $\mu_{i}=\lambda_{i}+\ell-i$, so subtracting one from each part of $\mu$ is the same as subtracting one from each part of $\lambda$. Thus, the partition $\left(\mu_{1}-1, \mu_{2}-1, \ldots, \mu_{\ell}-1\right)$ is associated to the partition $\lambda-1$. Similarly, the partition $\left(\mu_{2}-1, \mu_{3}-1, \ldots, \mu_{\ell}-1\right)$ is associated to the partition $\lambda-h$. The second term in (6.6) can be written

$$
-\mu_{1} \sum_{k=0}^{\infty}(-1)^{k-1} w_{k-1}\left(\mu_{1}-1, \mu_{2}-1, \ldots, \mu_{\ell}-1\right)=-\mu_{1} \sum_{k=0}^{\infty}(-1)^{k} w_{k}\left(\mu_{1}-1, \mu_{2}-1, \ldots, \mu_{\ell}-1\right),
$$

because by convention $w_{-1}=0$. Thus (6.6) becomes

$$
\eta_{\lambda}^{\prime}=\eta_{\lambda-h}^{\prime}-\mu_{1} \eta_{\lambda-1}^{\prime}
$$

Now, $\mu_{1}=\lambda_{1}+\ell-1=h$. Moreover, $|\lambda|=n$ implies that $|\lambda-h|=n-h$ and $|\lambda-1|=n-\ell$. Hence, from (6.5) and (6.8)

$$
\begin{aligned}
(-1)^{n} \eta_{\lambda} & =(-1)^{n-h} \eta_{\lambda-h}-(-1)^{n-\ell} h \eta_{\lambda-1} \\
& =(-1)^{n-h}\left(\eta_{\lambda-h}+(-1)^{\lambda_{1}} h \eta_{\lambda-1}\right),
\end{aligned}
$$

from which the stated recurrence follows. Finally, Equation (6.1) and the fact that $w_{0}=1$ gives $\eta_{\emptyset}=1$. (Note that we have implicitly used the stabilization property of complete factorial symmetric functions (Theorem 5.2).) 
Theorem 6.5 allows us to compute the eigenvalues of the derangement graph corresponding to certain simple shapes. For example, for hook shapes we have

Corollary 6.6. Let $\lambda=j 1^{n-j}$ denote the hook shape with first part $j$ and remaining parts 1. Then for $1 \leq j \leq n$,

$$
\eta_{j 1^{n-j}}=(-1)^{n}\left(1+(-1)^{j} n D_{j-1}\right)
$$

Proof. From Theorem 6.5

$$
\eta_{j 1^{n-j}}=(-1)^{n}\left(\eta_{\emptyset}+(-1)^{j} \cdot n \cdot \eta_{j-1}\right)
$$

Furthermore, either from the recurrence itself or directly from Theorem 2.1 we see that $\eta_{r}=\sum_{\sigma \in \mathcal{D}_{r}} 1=D_{r}$.

Remark 6.7. This result also follows with a little work from the properties of derangement numbers given in Lemma 4.1 and a result of Okazaki ([15]; see [20], Exercise 7.63, p. 469).

\section{Proof of the Conjecture}

Theorem 7.1. Conjecture 1.1 holds for all $\lambda$ with $n \geq 4$, and, moreover, for $n \geq 5$ the standard representation gives the unique minimum eigenvalue.

The proof is divided into four parts. First we show that the maximum eigenvalue is obtained by the trivial representation. Next, we show the conjecture holds for hooks, then that it holds for all near hooks, that is, partitions of the form $\lambda=j 21^{n-j-2}$ where $2 \leq j \leq n-2$. Finally, we show that it holds for all other partitions. First note that, by direct computation using Theorem 6.5 , we have, for $n=4: \eta_{1^{4}}=-3, \eta_{21^{2}}=1, \eta_{2^{2}}=$ $3, \eta_{31}=-3, \eta_{4}=9$. Clearly, in this case the least eigenvalue is achieved by the standard representation $\lambda=31$, but it is not unique, as the minimum is also realized by the alternating representation.

Lemma 7.2. The maximum eigenvalue of the derangement graph $\Gamma_{n}$ is $\eta_{n}=D_{n}$.

Proof. This can be shown using Theorem 6.5, but it follows most easily from the fact that $k$ is the largest eigenvalue of any regular graph of degree $k$. (See, e.g., [2], Proposition 3.1.)

Lemma 7.3. Conjecture 1.1 holds for hooks.

Proof. From (6.11)

$$
\eta_{(n-1,1)}=(-1)^{n}\left(1+(-1)^{n-1} n D_{n-2}\right)=(-1)^{n}-n D_{n-2},
$$


$\mathrm{SO}$

$$
\begin{aligned}
\alpha & :=\eta_{j 1^{n-j}}-\eta_{(n-1,1)}=(-1)^{n}\left(1+(-1)^{j} n D_{j-1}\right)-(-1)^{n}+n D_{n-2} \\
& =n\left(D_{n-2}+(-1)^{n+j} D_{j-1}\right) .
\end{aligned}
$$

We must show that $\alpha>0$ for $j<n-1$. (By Lemma 7.2 we already know the trivial representation corresponding to $j=n$ is positive-indeed, it is the largest eigenvalue.) If $n+j$ is even then $\alpha>0$ and there is nothing to prove, so assume $n+j$ is odd. As $n \geq 5$, $D_{n-2}>D_{j-1}$ for all $1 \leq j \leq n-2$ by Lemma 4.1 (ii), whence it follows that $\alpha>0$.

Lemma 7.4. Conjecture 1.1 holds for near hooks.

Proof. Applying Theorem 6.5 twice gives

$$
\begin{aligned}
\eta_{j 21^{n-j-2}} & =(-1)^{n-1}\left(\eta_{1}+(-1)^{j}(n-1) \eta_{(j-1,1)}\right) \\
& =(-1)^{n+j-1}(n-1) \eta_{(j-1,1)} \\
& =(-1)^{n-1}(n-1)\left(1+(-1)^{j-1} j D_{j-2}\right) \\
& =(n-1)\left((-1)^{n-1}+(-1)^{n+j} j D_{j-2}\right) .
\end{aligned}
$$

Thus, from (7.1) and (7.2),

$$
\begin{aligned}
\beta & :=\eta_{j 21^{n-j-2}}-\eta_{(n-1,1)} \\
& =(n-1)\left((-1)^{n-1}+(-1)^{n+j} j D_{j-2}\right)+n D_{n-2}+(-1)^{n-1} \\
& =n D_{n-2}+(-1)^{n+j}(n-1) j D_{j-2}+(-1)^{n-1} n .
\end{aligned}
$$

We must show $\beta>0$. But, regardless of the parity of $n+j$,

$$
\beta \geq n D_{n-2}-(n-1)(n-2) D_{n-4}+(-1)^{n-1} n,
$$

because $D_{j-2} \leq D_{n-4}$ for $j \leq n-2$ and $n \geq 5$ by Lemma 4.1 (ii). Using Lemma 4.1 (iii) we can write

$$
D_{n-2}=(n-2) D_{n-3}+(-1)^{n-2}=(n-2)(n-3) D_{n-4}+(n-2)(-1)^{n-3}+(-1)^{n-2},
$$

SO

$$
\begin{aligned}
\beta & \geq[n(n-2)(n-3)-(n-1)(n-2)] D_{n-4}+n(n-2)(-1)^{n-1} \\
& =[n-2]\left[\left(n^{2}-4 n+1\right) D_{n-4}+(-1)^{n-1} n\right]>0
\end{aligned}
$$

because both terms in square brackets are positive for $n \geq 5$.

Proof (of Theorem 7.1). We have already verified the result in the case $n=4$, so assume $n \geq 5$. Now let $\lambda \vdash n$. By Lemmas 7.3 and 7.4 we may assume $\lambda$ is neither a hook (in which case we would have $n=h$ ), nor a near hook (in which case we would have 
$n=h+1$ ). So we may safely assume $n \geq h+2$ and $h>\ell \geq 2$. Then we get the following chain of equalities and inequalities:

$$
\begin{aligned}
\left|\eta_{\lambda}\right| & =\left|\eta_{\lambda-h}+(-1)^{\lambda_{1}} h \eta_{\lambda-1}\right| & & (\text { Theorem 6.5) } \\
& \leq\left|\eta_{\lambda-h}\right|+h\left|\eta_{\lambda-1}\right| & & \text { (Lemma 7.2) } \\
& \leq D_{n-h}+h D_{n-\ell} & & \text { (Lemma 4.1 (ii) and } h>\ell) \\
& <(1+h) D_{n-\ell} & & \\
& \leq(n-1) D_{n-\ell} & & \\
& \leq(n-1) D_{n-2} & & \\
& =D_{n-1}+(-1)^{n} & & \text { Lemma 4.1 (iii)) } \\
& \leq D_{n-1}+1 & & \text { Lemma 4.1 (iii)) } \\
& <D_{n-1}+D_{n-2} & & D_{n} \\
& =\frac{\text { Equation }(2.2)) .}{n-1} & &
\end{aligned}
$$

\section{References}

[1] L. Babai, "Spectra of Cayley Graphs", J. Comb. Th. B 27 (1979), 180-189.

[2] N. Biggs, Algebraic Graph Theory, 2nd Edition (Cambridge University Press, Cambridge, 1993).

[3] P.J. Cameron and C.Y. Ku, "Intersecting Families of Permutations", Eur. J. Comb. 24 (2003), 881-890.

[4] W.Y.C. Chen and J.D. Louck, "The Factorial Schur Function", J. Math. Phys. 34 (1993), 4144-4160.

[5] M. Deza and P. Frankl, "On the Maximum Number of Permutations with Given Maximal or Minimal Distance", J. Combin. Th. A 22 (1977), 352-360.

[6] P. Diaconis and M. Shahshahani, "Generating a Random Permutation with Random Transpositions", Zeit. für Wahrscheinlichkeitstheorie 57 (1981), 159-179.

[7] R.B. Eggleton and W.D. Wallis, "Problem 86: Solution I", Math. Mag. 58 (1985), 112-113.

[8] C. Godsil, "Interesting Graphs and Their Colourings", April 3, 2006 version, unpublished lecture notes available at http://quoll.uwaterloo.ca/pstuff/colours.pdf.

[9] C. Godsil and K. Meagher, "A New Proof of the Erdős-Ko-Rado Theorem for Intersecting Families of Permutations", preprint available at http://front.math.ucdavis.edu/0710.2109. 
[10] D. Goldschmidt, Group Characters, Symmetric Functions, and the Hecke Algebra (American Mathematical Society, Providence, 1993).

[11] C. Ku and T. Wong, "Intersecting Families in the Alternating Group and Direct Product of Symmetric Groups", Elect. J. Combin. 14(1) (2007), \#R25.

[12] B. Larose and C. Malvenuto, "Stable Sets of Maximal Size in Kneser-Type Graphs", Europ. J. Combin. 25(5) (2004), 657-673.

[13] I.G. Macdonald, Symmetric Functions and Hall Polynomials, 2nd Edition (Oxford University Press, Oxford, 1995).

[14] M. Newman, "Independent Sets and Eigenspaces" (Thesis, University of Waterloo, 2004), available at http://www.maths.qmul.ac.uk/ mn/papers/indandeig.pdf

[15] S. Okazaki, PhD Thesis, Massachusetts Institute of Technology, 1992.

[16] A. Okounkov and G. Olshanski, "Shifted Schur Functions", Algebra i Analiz. 9 (1977), 73-146 (Russian). English translation: St. Petersburg Math. J. 9 (1998), 239-300. Available at http://arxiv.org/abs/q-alg/9605042.

[17] M. Ram Murty, "Ramanujan Graphs", J. Ramanujan Math. Soc. 18 (2003), 1-20.

[18] D. J. Rasmussen and C. D. Savage, "Hamilton-Connected Derangement Graphs on $S_{n}{ }^{\prime}$, Discrete Math. 133 (1994), 217-223.

[19] J.P. Serre, Linear Representations of Finite Groups (Springer-Verlag, New York, 1977).

[20] R.P. Stanley, Enumerative Combinatorics, Volume 2 (Cambridge University Press, Cambridge, 1999).

[21] R.P. Stanley, Enumerative Combinatorics, Volume 1 (Wadsworth \& Brooks/Cole, Monterey, CA, 1986).

[22] L. Verde-Star, "Divided Differences and Combinatorial Identities", Stud. Appl. Math. 85 (1991), 215-242. 